\title{
Robust Receding Horizon Control with Free Control Moves for Polytopic Parametric Uncertainty Systems Subject to Multiple Input Delays
}

\author{
Jiandong Yang $\mathbb{D}^{1}{ }^{1}$ Yuanli Cai $\mathbb{D},{ }^{1}$ and Baocang Ding $\mathbb{D}^{2}$ \\ ${ }^{1}$ Department of Automation, School of Electronic and Information Engineering, Xi'an Jiaotong University, Xi'an 710049, China \\ ${ }^{2}$ College of Automation, Chongqing University of Posts and Telecommunications, Chongqing 400065, China
}

Correspondence should be addressed to Yuanli Cai; ylicai@mail.xjtu.edu.cn and Baocang Ding; baocang.ding@gmail.com

Received 5 November 2019; Revised 19 January 2020; Accepted 25 January 2020; Published 22 April 2020

Academic Editor: Jose Vicente Salcedo

Copyright ( 12020 Jiandong Yang et al.This is an open access article distributed under the Creative Commons Attribution License, which permits unrestricted use, distribution, and reproduction in any medium, provided the original work is properly cited.

A robust receding horizon control (RHC) with free control moves is applied to polytopic parametric uncertainty systems with multiple input delays and unstable system matrices. A difficulty in the previous robust RHC work is that the free control moves are unsuitable for the system with input time delay, which is overcome in this paper by the design based on the augmented state. As a result, the synthesis of local control based on augmented feedback is given offline to alleviate the online computation burden. The free control moves before the augmented feedback are the online decision variables, which are solved by minimizing a sequence of nonnegative scalars online. The recursive feasibility is guaranteed by adopting the augmented state space equation. By adjusting the robust positively invariant set, the stability of the closedloop system is guaranteed. Simulation results demonstrate that the proposed algorithm improves the control performance effectively.

\section{Introduction}

Receding horizon control (RHC) is known for the capacity to deal with the multivariable constraint by the receding horizon optimization. It has obtained a lot of attention in academic [1-6], and the control policy has been applied to many actual systems such as multiagent systems [7], chemical engineering [8], and hybrid electric vehicles [9].

Since modeling errors cannot be avoided in the practical systems, the uncertainties and disturbance usually exist in the system model. There are many results focused on the robust control for the system with the uncertainty and disturbance $([10-12])$. In particular, the robust RHC is a hot topic in recent years (see, e.g., [13-16]). A lot of significant results for the state feedback RHC with uncertainty have appeared in the literature ([17-20]). Besides, time delays usually exist in dynamic systems $([10,21])$. The presence of time delays often leads to oscillation and instability of the closed-loop system. The controller design for the time delay systems is often crucial for the system operation. A lot of researchers have devoted to designing a robust MPC controller for the system with uncertainties and time delay. In [22], robust RHC for the model with polytopic parametric uncertainty and one state delay is studied. The local control is a memoryless feedback. The recursive feasibility is well guaranteed under the proper assumption of terminal weighting matrices. The stability of the system is proved by choosing the Lyapunov function and feedback gain properly. In [23], for the positive systems with polytopic parametric uncertainty and multiple state delays, a robust RHC scheme is proposed. A linear copositive Lyapunov function and a cone invariant set are used to prove the stability of the system. In [24], a robust RHC for the system with state and random input delays is studied. The 
robust RHC for the systems with one input delay and more than one state delay is first proposed in [25], but the implementation details are not given. In [26], the implementation details are rectified. If the local feedback is adopted the same as [25], the system matrices are required to be asymptotically stable.

For the robust RHC, many researchers have made effort to obtain a better control performance. In [25], the infinite horizon robust $\mathrm{RHC}$ is parameterized into a single feedback gain $K$. One free control move is added before $K$ in [27]. As a result, the region of attraction is enlarged, and a better control performance is achieved. In [28], the approach with $N \geq 1$ free control moves is adopted. First, the local feedback gain $K$ is calculated offline, so the online computation burden is reduced significantly. At each time $k$, the predictive control input is defined as $u_{i}(k)=K x_{i}(k)+v_{i}(k)$, where $v_{i}(k)$ is the free perturbation being the online decision variable. This approach not only guarantees the closed-loop stability but also can get a bigger region of attraction compared with the approach of one free control move. Hence, a better performance and a wider applicability can be achieved. For the state feedback robust RHC, the successful applications of this approach have been witnessed, e.g., [29], $N$ free control moves are applied to the systems with norm-bounded uncertainty. In [19], by incorporating more uncertain system information, an improved free control move approach is proposed. In [26], the free control move approach is extended to the systems with uncertainty and multiple input delays. By modifying the local feedback, this approach can be applied to the system with unstable matrices. However, this approach make the optimization problem lose the recursive feasibility. This motivates us to design a new RHC synthesis control scheme to restore the recursive feasibility. The contribution of this paper will be highlighted as follows:

(1) The local control adopts augmented state feedback, where the augmented state contains past inputs and states, and the heavy computation burden incurred by augmented feedback is removed offline

(2) The recursive feasibility and closed-loop stability are guaranteed, even when the free control moves are introduced before the augmented state, since the augmented state space equation is used

(3) The unstable system matrices can be handled by adopting the augmented state space model, which is the least conservative treatment of state delays and input delays, whether the free control moves are introduced or not

The organization of our work is summarized as follows. In Section 2, the problem statement is given. The offline robust controller synthesis for the multiple time delay systems is presented in Section 3. A general robust positively invariant set is proposed which serves as the terminal invariant set. In Section 4, the receding horizon control policy with free control moves is given. Simulation results demonstrate that receding horizon control with free control moves is effective in Section 5. Finally, a conclusion of the paper is stated.

Notations. $\|x\|_{M}^{2}:=x^{T} M x$ and $\varepsilon_{M}:=\left\{x \mid x^{T} M x \leq 1\right\}$, where $x$ is a vector and matrix $M>0$. For the vectors $s_{1}$ and $s_{2}$, let $\left[s_{1} ; s_{2}\right]:=\left[s_{1}^{T}, s_{2}^{T}\right]^{T}$. For the matrix $T=\left[\begin{array}{cc}T_{11} & \star \\ T_{21} & T_{22}\end{array}\right]$, the symbol $\star$ represents a symmetric matrix of $T_{21} \cdot x_{i}(k)$ is the $i$-step ahead predicted state of $x(k)$.

\section{Problem Statement}

Consider a polytopic parametric uncertainty system with multiple time delays:

$$
x(k+1)=\sum_{\mu=0}^{m} A_{\mu}(k) x(k-\mu)+\sum_{\tau=0}^{n} B_{\tau}(k) u(k-\tau),
$$

where $x \in \mathbb{R}^{p}$ is measurable state, $u \in \mathbb{R}^{q}$ is the control input, $\mu \geq 0, \tau \geq 0$, and $m$ and $n$ are positive integers.

The following assumptions are given.

Assumption 1. The polytopic parametric uncertainty matrices $A_{\mu}$ and $B_{\tau}$ belong to a polytope $\Sigma$, i.e.,

$$
\begin{aligned}
& {\left[A_{0}|\cdots| A_{m}\left|B_{1}\right| \cdots \mid B_{n}\right] \in \Sigma, } \\
\Sigma & =\operatorname{Co}\left\{\left[A_{0}^{(l)}|\cdots| A_{m}^{(l)}\left|B_{0}^{(l)}\right| \cdots \mid B_{n}^{(l)}\right], \quad l \in \mathbb{D}\right\},
\end{aligned}
$$

where $\mathbb{D}:=\{1,2, \ldots, L\} .\left[A_{0}^{(l)}|\cdots| A_{m}^{(l)}\left|B_{0}^{(l)}\right| \cdots \mid B_{n}^{(l)}\right]$ are the vertices of $\Sigma$.

Assumption 2. The multiple time delay system (1) satisfies the following physical constraints:

$$
\begin{gathered}
-\nu \leq u(k) \leq \nu, \\
-\phi \leq \Psi x(k) \leq \phi,
\end{gathered}
$$

where $\quad v=\left[v_{1}, v_{2}, \ldots, v_{q}\right]^{\mathrm{T}}, v_{s} \geq 0, s=1, \ldots, q . \quad \phi=\left[\phi_{1}\right.$, $\left.\phi_{2}, \ldots, \phi_{p}\right]^{\mathrm{T}}, \phi_{j} \geq 0, j=1, \ldots, p . \Psi \in \mathbb{R}^{p \times p}$.

A local feedback control is designed:

$$
u(k)=\sum_{\mu=0}^{m} F_{\mu}(k) x(k-\mu)+\sum_{\tau=1}^{n} H_{\tau}(k) u(k-\tau)
$$

where $\left\{F_{\mu}, \mu=0,1, \ldots, m\right\}$ and $\left\{H_{\tau}, \tau=1,2, \ldots, n\right\}$ are the feedback gains to be designed.

Define $\quad \bar{x}:=[x(k) ; x(k-1) ; \ldots ; x(k-m)], \quad \bar{u}:=[u$ $(k-1) ; \ldots ; u(k-n)]$, and $\xi(k):=[\bar{x} ; \bar{u}]$. To compensate 
the multiple time delays of the system, an augmented state is established. Based on systems (1) and (5), one obtains the following augmented model:

$$
\xi(k+1)=\Gamma(k) \xi(k)
$$

where

$$
\begin{aligned}
& \Gamma=\left[\begin{array}{cc}
\Gamma_{1} & \Gamma_{2} \\
\widetilde{F} & \widetilde{H}
\end{array}\right], \\
& \Gamma_{1}=\left[\begin{array}{cc}
\Phi_{1} & A_{m} \\
I_{m \times m} & 0_{m \times 1}
\end{array}\right], \\
& \Gamma_{2}=\left[\begin{array}{cc}
\Phi_{2} & B_{n} \\
0_{n \times(n-1)} & 0_{n \times 1}
\end{array}\right] \text {, } \\
& \widetilde{F}=\left[\begin{array}{cc}
\Phi_{3} & F_{m} \\
0_{m \times m} & 0_{m \times 1}
\end{array}\right] \text {, } \\
& \tilde{H}=\left[\begin{array}{cc}
\Phi_{4} & H_{q} \\
I_{m \times n-1} & 0_{m \times 1}
\end{array}\right], \\
& \Phi_{1}=\left[\begin{array}{llll}
\mathbf{A}_{0} & \mathbf{A}_{1} & \cdots & \mathbf{A}_{m-1}
\end{array}\right] \text {, } \\
& \mathbf{A}_{\mu}=A_{\mu}+B_{0} F_{\mu}, \quad \mu=0,1, \ldots, m, \\
& \Phi_{2}=\left[\begin{array}{llll}
\mathbf{B}_{1} & \mathbf{B}_{2} & \cdots & \mathbf{B}_{n-1}
\end{array}\right], \\
& \mathbf{B}_{\tau}=B_{\tau}+B_{0} H_{\tau}, \quad \tau=1,2, \ldots, n, \\
& \Phi_{3}=\left[\begin{array}{llll}
F_{0} & F_{1} & \cdots & F_{m-1}
\end{array}\right], \\
& \Phi_{4}=\left[\begin{array}{llll}
H_{1} & H_{2} & \cdots & H_{n-1}
\end{array}\right] .
\end{aligned}
$$

Remark 1. It is worth noting that a polytopic parametric uncertainty system with multiple time delay is all inclusive in the mathematical model.

(1) When all values of $\mu$ and $\tau$ are equal to zero, system (1) is translated to the system without time delay:

$$
x(k+1)=A_{0}(k) x(k)+B_{0}(k) u(k) .
$$

(2) When the values of $\mu$ and $\tau$ are 0 or 1 , system (1) is translated to the system with state delay or input delay. For example,

$$
\begin{aligned}
& x(k+1)=A_{\mu}(k) x(k-\mu)+B_{0}(k) u(k), \\
& x(k+1)=A_{\tau}(k) x(k)+B_{\tau}(k) u(k-\tau), \\
& x(k+1)=A_{\mu}(k) x(k-\mu)+B_{\tau}(k) u(k-\tau) .
\end{aligned}
$$

(3) The mathematical model in this paper is a more general case for the dynamic system.

\section{Offline Robust Controller Synthesis}

Given matrices $\mathbb{Q}>0$ and $\mathscr{R}>0$, the following cost function is considered:

$$
J=\sum_{k=0}^{\infty}\left(x(k)_{\mathscr{Q}}^{2}+\|u(k)\|_{\mathscr{R}}^{2}\right) .
$$

We will design a local feedback (4) by minimizing $J$, for system (6) satisfying constraints (3) and (4).

The statement is summarized into the following optimization problem:

$$
\begin{array}{ll}
\min _{K} & \max _{\star} J \\
\text { s.t. } & (3),(4),
\end{array}
$$

where

$$
\begin{aligned}
K & =\left[F_{0} \cdots F_{m} H_{1} \cdots H_{n}\right], \\
\star & =\left[A_{0}\left|A_{1}\right| \cdots\left|A_{m}\right| B_{1}\left|B_{2}\right| \cdots \mid B_{n}\right] \in \Sigma .
\end{aligned}
$$

Consider the following Lyapunov function for system (6):

$$
V(\xi(k))=\xi(k)^{T} \widetilde{Q}^{-1} \xi(k),
$$

where

$$
\begin{aligned}
\widetilde{Q} & =\left[\begin{array}{cc}
Q & \star \\
R & M
\end{array}\right], \\
Q & =\left[\begin{array}{cccc}
Q_{00} & Q_{01} & \cdots & Q_{0, m} \\
Q_{10} & Q_{11} & \cdots & Q_{1, m} \\
\vdots & \vdots & \ddots & \vdots \\
Q_{m, 0} & Q_{m, 1} & \cdots & Q_{m, m}
\end{array}\right], \\
M & =\left[\begin{array}{cccc}
M_{11} & M_{12} & \cdots & M_{1, n} \\
M_{21} & M_{22} & \cdots & M_{2, n} \\
\vdots & \vdots & \ddots & \vdots \\
M_{n, 1} & M_{n, 2} & \cdots & M_{n, n}
\end{array}\right], \\
R & =\left[\begin{array}{cccc}
R_{10} & R_{11} & \cdots & R_{1, m} \\
R_{20} & R_{21} & \cdots & R_{2, m} \\
\vdots & \vdots & \ddots & \vdots \\
R_{n, 0} & R_{n, 1} & \cdots & R_{n, m}
\end{array}\right] .
\end{aligned}
$$

The following inequality is imposed: 


$$
V(\xi(k+1))-V(\xi(k)) \leq-\frac{1}{\lambda}\left(\|x(k)\|_{\mathscr{Q}}^{2}+\|u(k)\|_{\mathscr{R}}^{2}\right),
$$

where $\lambda$ is a scalar, then the robust stability of system (6) holds.

Let the Lyapunov function $V(\xi(k)) \leq 1$, which can be described by

$$
\xi(k) \in \mathcal{E}_{\widetilde{Q}^{-1}} .
$$

By summing (15) from $k=0$ to $k=\infty$, one obtains

$$
\max _{\star} J \leq \lambda V(\xi(0)) \leq \lambda .
$$

Therefore, the offline optimization problem (11) is rewritten as

$$
\begin{array}{ll}
\min _{K} & \lambda \\
\text { s.t. } & (3),(4),(15),(16) .
\end{array}
$$

The optimization problem (18) is nonconvex. It will be transformed into the convex optimization problem by LMI technique ([25]).

Theorem 1. If there exist a scalar $\lambda>0$ and matrices $T_{\mu}$, $\mu \in\{0,1, \ldots, m\}, S_{\tau}, \tau \in\{1, \ldots, n\}, \widetilde{Q}>0, \Gamma, \Theta$, such that the following inequalities

$\min \lambda$,

$$
\begin{aligned}
& \text { s.t. }\left[\begin{array}{ccc}
\widetilde{Q} & \star & \star \\
\Upsilon^{(l)} & \widetilde{Q} & \star \\
\Omega & 0 & \lambda I
\end{array}\right] \geq 0, \quad l \in \mathbb{D}, \\
& \quad\left[\begin{array}{cc}
1 & \star \\
\xi(k) & \widetilde{Q}
\end{array}\right] \geq 0, \\
& {\left[\begin{array}{cc}
\widetilde{Q} & \star \\
\widetilde{K} & \Xi
\end{array}\right] \geq 0, \quad \Xi_{s, s} \leq v^{2}, s=1, \ldots, q,} \\
& {\left[\begin{array}{cc}
\widetilde{Q} & \star \\
\widetilde{\Upsilon}^{(l)} & \Theta
\end{array}\right] \geq 0, \quad \Theta_{j, j} \leq \phi^{2}, j=1, \ldots, p,}
\end{aligned}
$$

where

$$
\begin{aligned}
\Upsilon & =\left[\begin{array}{cc}
\Upsilon_{1} & \Upsilon_{2} \\
\Upsilon_{3} & \Upsilon_{4}
\end{array}\right], \\
\Upsilon_{1} & =\left[\begin{array}{cccc}
\widetilde{A}_{0} & \widetilde{A}_{1} & \cdots & \widetilde{A}_{m} \\
Q_{00} & Q_{01} & \cdots & Q_{0, m} \\
Q_{10} & Q_{11} & \cdots & Q_{1, m} \\
\vdots & \vdots & \ddots & \vdots \\
Q_{m-1,0} & Q_{m-1,1} & \cdots & Q_{m-1, m}
\end{array}\right],
\end{aligned}
$$

$$
\widetilde{A}_{\mu}=\sum_{h=0}^{m} A_{h} Q_{h, \mu}+\sum_{g=1}^{n} B_{g} R_{g, \mu}+B_{0} T_{\mu}
$$$$
\Upsilon_{2}=\left[\begin{array}{cccc}
\widetilde{B}_{1} & \widetilde{B}_{2} & \cdots & \widetilde{B}_{n} \\
R_{10}^{T} & R_{2,0}^{T} & \cdots & R_{n, 0}^{T} \\
R_{11}^{T} & R_{2,1}^{T} & \cdots & R_{n, 1}^{T} \\
\vdots & \vdots & \ddots & \vdots \\
R_{1, m-1}^{T} & R_{2, m-1}^{T} & \cdots & R_{n-1, m}^{T}
\end{array}\right],
$$$$
\widetilde{B}_{\tau}=\sum_{h=0}^{m} A_{h} R_{\tau, h}^{T}+\sum_{g=1}^{n} B_{g} M_{\tau, g}+B_{0} S_{\tau}
$$$$
\Upsilon_{3}=\left[\begin{array}{cccc}
T_{0} & T_{1} & \cdots & T_{m} \\
R_{10} & R_{11} & \cdots & R_{1, m} \\
R_{20} & R_{21} & \cdots & R_{2, m} \\
\vdots & \vdots & \ddots & \vdots \\
R_{n-1,0} & R_{n-1,1} & \cdots & R_{n-1, m}
\end{array}\right],
$$$$
T_{\mu}=\sum_{h=0}^{m} F_{h} Q_{h, \mu}+\sum_{g=1}^{n} H_{g} R_{g, \mu}^{T}
$$$$
\Upsilon_{4}=\left[\begin{array}{cccc}
S_{1} & S_{2} & \cdots & S_{n} \\
M_{11} & M_{12} & \cdots & M_{1, n} \\
M_{21} & M_{22} & \cdots & M_{2, n} \\
\vdots & \vdots & \ddots & \vdots \\
M_{n-1,1} & M_{n-1,2} & \cdots & M_{n-1, n}
\end{array}\right] \text {, }
$$

$$
\begin{aligned}
& S_{\tau}=\sum_{h=0}^{m} F_{h} R_{\tau, h}^{T}+\sum_{g=1}^{n} H_{g} M_{\tau, g} \text {, } \\
& \Omega=\left[\begin{array}{ll}
\Omega_{1} & \Omega_{2} \\
\Omega_{3} & \Omega_{4}
\end{array}\right], \\
& \Omega_{1}=\left[\begin{array}{llll}
\Omega^{1 / 2} Q_{00} & \cdots & Q^{1 / 2} Q_{0, m}
\end{array}\right], \\
& \Omega_{2}=\left[\begin{array}{lll}
\Omega^{1 / 2} R_{10}^{T} & \cdots & Q^{1 / 2} R_{n, 0}^{T}
\end{array}\right], \\
& \Omega_{3}=\left[\begin{array}{llll}
\mathscr{R}^{1 / 2} T_{1} & \cdots & \mathscr{R}^{1 / 2} T_{m}
\end{array}\right], \\
& \Omega_{4}=\left[\begin{array}{llll}
\mathscr{R}^{1 / 2} S_{1} & \cdots & \mathscr{R}^{1 / 2} S_{n}
\end{array}\right], \\
& \tilde{\Upsilon}=\Psi G \Upsilon \widetilde{Q} \text {, } \\
& G=\left[\begin{array}{ll}
I_{p \times p} & 0
\end{array}\right] \text {, } \\
& \widetilde{K}=\left[\begin{array}{lllllll}
T_{0} & T_{1} & \cdots & T_{m} & S_{1} & \cdots & S_{n}
\end{array}\right],
\end{aligned}
$$

then controller (5) stabilizes system (6) satisfying physical constraints (3) and (4), where the controller gain $K=\widetilde{K} \widetilde{Q}^{-1}$.

Proof. Substituting (6) into (15), one has

$$
\xi(k)^{T}\left(\widetilde{\widetilde{Q}}^{-1}-\Gamma^{T}(k) \widetilde{\mathscr{Q}}^{-1} \Gamma(k)-\frac{1}{\lambda G^{T} \mathscr{R} G}-\frac{1}{\lambda K^{T} \mathscr{R} K}\right) \xi(k) \geq 0 .
$$
has

Removing $\xi(k)$ and applying the Schur complement, one 


$$
\left[\begin{array}{ccc}
\widetilde{Q}^{-1} & \star & \star \\
\Gamma & \widetilde{Q} & \star \\
\widetilde{\Omega} & 0 & \lambda I
\end{array}\right] \geq 0,
$$

where $\widetilde{\Omega}=\left[\mathscr{R}^{1 / 2} K ; \mathscr{Q}^{1 / 2} G\right]$. Pre- and postmultiplying inequality (26) by $\operatorname{diag}\{\widetilde{Q}, I, I, I\}$ and its transposition, it yields

$$
\left[\begin{array}{ccc}
\widetilde{Q} & \star & \star \\
\Upsilon & \widetilde{Q} & \star \\
\Omega & 0 & \lambda I
\end{array}\right] \geq 0
$$

Note that the matrix $\Upsilon$ is the convex combination of matrices $\Upsilon^{(l)}, l \in \mathbb{D}$. If (20) holds, then (27) is true.

Obviously, applying the Schur complement, it is shown that $\xi(k) \in \varepsilon_{\widetilde{Q}^{-1}}$ is equivalent to (21).

We deal with the input constraint (3) and state constraint (4) by virtue of $\xi(k) \in \varepsilon_{\widetilde{\Omega}^{-1}}$.

Denote $\eta_{i}=[0, \underbrace{I}_{s-\text { th }}, 0]_{1 \times q}$. Then,

$$
\begin{aligned}
& \max _{k \geq 0}\left|\eta_{s} u(k)\right| \\
& =\max _{k \geq 0}\left|\eta_{s} K \xi(k)\right| \\
& \leq \max _{k \geq 0}\left\|\eta_{s} K \widetilde{Q}^{1 / 2}\right\|\left\|\widetilde{\mathbb{Q}}^{-1 / 2} \xi(k)\right\| \\
& \leq \max _{k \geq 0}\left\|\eta_{s} K \widetilde{Q}^{1 / 2}\right\| .
\end{aligned}
$$

If there exists a matrix $\Xi=\Xi^{T}$ and $\Xi_{s s} \leq v_{s}^{2}, s=1, \ldots, q$, such that

$$
\Xi-K^{T} \widetilde{Q} K \geq 0,
$$

then (28) holds.

Applying the Schur complement to the abovementioned inequality, one has

$$
\left[\begin{array}{cc}
\widetilde{Q}^{-1} & \star \\
K & \Xi
\end{array}\right] \geq 0, \quad \Xi_{s, s} \leq \nu^{2}, s=1, \ldots, q .
$$

Pre- and postmultiplying inequality (30) by $\operatorname{diag}\{\widetilde{Q}, I\}$ and its transposition, the abovementioned inequality is equivalent to (22). Therefore, input constraint (3) is guaranteed by (22).

$$
\begin{aligned}
& \text { Denote } \zeta_{j}=[0, \underbrace{I, 0]_{1 \times p} . \text { Then, }}_{j \text {-th }} \\
& \qquad \max _{k \geq 0}\left|\zeta_{j} \Psi x(k+1)\right| \\
& =\max _{k \geq 0}\left|\zeta_{j} \Psi G \xi(k+1)\right| \\
& =\max _{k \geq 0}\left|\zeta_{j} \Psi G \Gamma(k) \xi(k)\right| \\
& =\max _{k \geq 0}\left\|\zeta_{j} G \Psi \Gamma(k) \widetilde{\mathbb{Q}}^{1 / 2} \widetilde{Q}^{-1 / 2} \xi(k)\right\| \\
& \leq \max _{k \geq 0}\left\|\zeta_{j} \Psi G \Gamma(k) \widetilde{\mathbb{Q}}^{1 / 2}\right\|\left\|\widetilde{\mathbb{Q}}^{-1 / 2} \xi(k)\right\| \\
& \leq \max _{k \geq 0}\left\|\zeta_{j} \Psi G \Upsilon(k) \widetilde{\mathbb{Q}}^{-1 / 2}\right\| .
\end{aligned}
$$

If there exists a matrix $\Theta=\Theta^{T}$ and $\Theta_{j, j} \leq \phi^{2}$, $j=1, \ldots, p$, such that

$$
\Theta-\bar{\Upsilon}^{T} \widetilde{Q} \widetilde{Y} \geq 0
$$

where $\bar{\Upsilon}=\Psi G \Gamma$, then (31) holds.

By virtue of the Schur complement to (32), one has

$$
\left[\begin{array}{cc}
\widetilde{Q}^{-1} & \star \\
\bar{\Upsilon} & \Theta
\end{array}\right] \geq 0, \quad \Theta_{j, j} \leq \phi^{2}, j=1, \ldots, p .
$$

Pre- and postmultiplying inequality (33) by $\operatorname{diag}\{\widetilde{Q}, I\}$ and its transposition, the abovementioned inequality is equivalent to

$$
\left[\begin{array}{cc}
\widetilde{Q} & \star \\
\widetilde{\Upsilon} & \Theta
\end{array}\right] \geq 0, \quad \Theta_{j, j} \leq \phi^{2}, j=1, \ldots, p .
$$

According to the convexity of the polytopic uncertainty matrix $\widetilde{Y}$, if (23) holds, (34) is guaranteed. Therefore, input constraint (4) is guaranteed by (23).

It is shown that if inequalities (20)-(23) are feasible, controller (5) can stabilize system (6).

In conclusion, (11) can be formulated as follows:

$$
\begin{array}{ll}
\min & \lambda \\
\text { s.t. } & (20),(21),(22),(23) .
\end{array}
$$

Remark 2. In [26], $Q=\operatorname{diag}\left\{Q_{00}, \ldots, Q_{m, m}\right\} \quad$ and $M=\operatorname{diag}\left\{M_{11}, \ldots, M_{n, n}\right\}$. Obviously, the case in this paper covers that in [26].

Remark 3. The local feedback gain can also be optimized online, but the computation burden is heavy. In this paper, the local feedback gain is designed offline.

\section{Robust Receding Horizon}

The following receding horizon control policy is considered:

$$
u_{s}(k)= \begin{cases}K \xi_{s}(k)+v_{s}(k), & s \in \mathbb{T}, \\ K \xi_{s}(k), & s \geq N,\end{cases}
$$

where $\mathbb{T}=\{0,1, \ldots, N-1\}$ and $K$ is calculated offline. A sequence of control moves $v_{s}(k)$ are the online decision variables.

Based on (36), the prediction model is represented as

$$
\xi_{s+1}(k)=\Gamma_{s}(k) \xi_{s}(k)+\Lambda_{s}(k) v_{s}(k),
$$

where $\Lambda=\left[\begin{array}{llll}B_{0} & \underbrace{0}_{p} & I & \underbrace{0}_{q-1}\end{array}\right]^{T}$.

4.1. The Online Optimization. At every time $k$, the following problem is solved to find the free control moves $v_{s}(k)$ :

$$
v_{s}(k)_{s=0}^{N-1}=\arg \min _{v_{s}(k)} \max _{\star} \sum_{s=0}^{\infty}\left(\left\|x_{s}(k)\right\|_{\mathscr{Q}}^{2}+\left\|u_{s}(k)\right\|_{\mathscr{R}}^{2}\right) .
$$

Let $J_{s}(k):=\left\|x_{s}(k)\right\|_{\mathscr{Q}}^{2}+\left\|u_{s}(k)\right\|_{\mathscr{R}}^{2}, s \in \mathbb{T}$, which is represented as the stage cost; $J_{\infty}(k):=\sum_{s=N}^{\infty}\left\|x_{s}(k)\right\|_{\mathscr{Q}}^{2}+\left\|u_{s}(k)\right\|_{\mathscr{R}}^{2}$, which is represented as the terminal cost. 
For handling the stage cost easily, a sequence of nonnegative scalars $\lambda_{s}(k)$ are introduced $[26,29]$, which satisfy

$$
J_{s}(k) \leq \lambda_{s}(k), \quad s \in \mathbb{T} .
$$

The robust positively invariant set $\varepsilon_{\widetilde{Q}^{-1}}$ is the terminal constraint set and $\xi_{s}(k) \in \varepsilon_{\widetilde{Q}^{-1}}$. The quadratic function $\bar{V}\left(\xi_{s}(k)\right)=\xi_{s}(k)^{T} \widetilde{Q}^{-1} \xi_{s}(k)$ satisfies

$$
\bar{V}\left(\xi_{s+1}(k)\right)-\bar{V}\left(\xi_{s}(k)\right) \leq \frac{-1}{\lambda}\left(\left\|x_{s}(k)\right\|_{\mathscr{Q}}^{2}+\left\|u_{s}(k)\right\|_{\mathscr{R}}^{2}\right) .
$$

By summing (40), from $s=N$ to $s=\infty$, one has

$$
J_{\infty}(k) \leq \lambda \xi_{N}(k)^{T} \widetilde{Q}^{-1} \xi_{N}(k) \leq \lambda(k) .
$$

Therefore, the online optimization problem is stated as follows:

$$
\begin{gathered}
v_{s}(k)_{s=0}^{N-1}=\arg \min \lambda+\sum_{s=0}^{N-1} \lambda_{s}, \\
\text { s.t. } \quad J_{s}(k) \leq \lambda_{s}, \\
\left\|\xi_{N}(k)\right\|_{\widetilde{Q}^{-1}}^{2} \leq 1, \\
v \leq u_{s}(k) \leq \nu, \\
-\phi \leq \Psi x_{s+1}(k) \leq \phi .
\end{gathered}
$$

The prediction of the augmented state $\xi_{s}(k)$ is used in the optimization problem. Since the polytopic parametric uncertainty exists in the system, the deterministic value of $\xi_{s}(k)$ cannot be given directly. However, a tightest set can be constructed which contains all possible $\xi_{s}(k)$. The following lemma will be proposed to calculate the tightest set. It is a generalization of "Lemma 1" in [30].

Lemma 1. Let $\mathbb{S}_{i}(k)=\operatorname{Co}\left\{\xi_{i}^{(r)}(k), r=1,2, \ldots, L^{i}\right\}$ be a convex set. Suppose $\xi_{i}(k) \in \mathbb{S}_{i}(k), \forall i>0$, then $\mathbb{S}_{i+1}(k)$ is the tightest set of system (37), which contains all possible future states $\xi_{i+1}(k)$, if $\xi_{i+1}^{(j)}(k), j=1,2, \ldots, L^{i+1}$, satisfy

$$
\xi_{i+1}^{(j)}(k)=\Gamma_{i}^{(l)}(k) \xi_{i}^{(m)}(k)+\Lambda_{i}^{(l)}(k) v_{i}(k),
$$

for all $l \in \mathbb{D}, m \in\left\{1, \ldots, L^{i}\right\}$.

By induction, one obtains

$$
\xi_{i+1}^{(j)}(k)=\Gamma^{\left(l_{i}, \ldots, l_{0}\right)} \xi(k)+\sum_{t=0}^{i} \Gamma^{\left(l_{i}, \ldots, l_{t+1}\right)} \Lambda^{\left(l_{t}\right)}(k) v_{t}(k),
$$

where $\quad \Gamma^{\left(l_{i}, \ldots, l_{q}\right)}=\prod_{t_{q}=q}^{i} \Gamma^{\left(l_{t_{q}}\right)}\left(k+t_{q}\right), \quad l_{0}, l_{1}, \ldots, l_{i} \in \mathbb{D}$. $j+1 \leq i$.

Proof. For $i=0$, one has

$$
\xi_{1}^{(j)}(k)=\Gamma^{\left(l_{0}\right)} \xi(k)+\Lambda^{\left(l_{0}\right)}(k) v_{0}(k) .
$$

Suppose $i=h$, (48) holds, i.e.,

$$
\xi_{h+1}^{(j)}(k)=\Gamma^{\left(l_{h}, \ldots, l_{0}\right)} \xi(k)+\sum_{t=0}^{h} \Gamma^{\left(l_{h}, \ldots, l_{t+1}\right)} \Lambda^{\left(l_{t}\right)}(k) v_{t}(k) .
$$

Substituting (47) into (50), one obtains

$$
\begin{aligned}
\xi_{h+2}^{(j)}(k) & =\Gamma_{h+1}^{\left(l_{h+1}\right)}(k) \xi_{h+1}^{(m)}(k)+\Lambda_{h+1}^{\left(l_{h+1}\right)}(k) v_{h+1}(k) \\
& =\Gamma^{\left(l_{h+1}, \ldots, l_{0}\right)} \xi(k)+\sum_{t=0}^{h+1} \Gamma^{\left(l_{h+1}, \ldots, l_{t+1}\right)} \Lambda^{\left(l_{t}\right)}(k) v_{h+1}(k) .
\end{aligned}
$$

Therefore, if (47) holds, the abovementioned lemma is true and (48) holds.

Lemma 2. There exist a sequence of scalars $\lambda_{s}(k)>0, s \in \mathbb{T}$, such that

(a) (43) is guaranteed by

$$
\left[\begin{array}{ccc}
\lambda_{s}(k) & \star & \star \\
G \xi_{s}^{(j)}(k) & Q^{-1} & \star \\
\left.\left.K \xi_{s}^{(j)}(k)\right)+v_{s}(k)\right) & 0 & \mathscr{R}^{-1}
\end{array}\right] \geq 0 ;
$$

(b) (44) is equivalent to

$$
\left[\begin{array}{cc}
1 & \star \\
\xi_{N}^{(j)}(k) & \widetilde{Q}
\end{array}\right] \geq 0
$$

(c) The physical constraints (45) and (46) are guaranteed by

$$
\begin{aligned}
-\nu & \leq K \xi_{s}^{(j)}(k)+v_{s}(k) \leq \nu, \\
- & =\psi(54) \\
& s \in\{0,1, \ldots, N-1\}, j \in\left\{1,2, \ldots, \xi_{s}^{(j)}(k) \leq \psi,\right.
\end{aligned}
$$

Proof. (a) Substituting

$$
\begin{aligned}
& x_{s}(k)=G \xi_{s}(k), \\
& u_{s}(k)=K \xi_{s}(k)+v_{s}(k),
\end{aligned}
$$

into (43), it yields

$$
\left\|G \xi_{s}(k)\right\|_{\mathscr{Q}}^{2}+\left\|K \xi_{s}(k)+v_{s}(k)\right\|_{\mathscr{R}}^{2} \leq \lambda_{s}(k) .
$$

Using Schur complements, (57) is transformed into

$$
\left[\begin{array}{ccc}
\lambda_{s}(k) & \star & \star \\
G \xi_{s}(k) & Q^{-1} & \star \\
K \xi_{s}(k)+v_{s}(k) & 0 & \mathscr{R}^{-1}
\end{array}\right] \geq 0,
$$


where $\xi_{s}(k) \in \mathcal{S}_{s}(k)$, the vertices of $\mathcal{S}_{s}(k)$ are represented by (48). If every vertex $\xi_{s}^{(j)}(k)$ satisfies (52), then (58) holds.

(b) In the same way, if (53) holds, the terminal constraint (44) is guaranteed.

(c) Before the receding horizon $N$, if (45) and (46) hold, (54) and (55) are satisfied, respectively.

Therefore, the online optimization problem (42) is reformulated as follows:

$$
\begin{array}{ll}
\min _{v_{s}(k)} & \sum_{s=0}^{N-1} \lambda_{s} \\
\text { s.t. } & (52)-(55) .
\end{array}
$$

\subsection{Recursive Feasibility and Stability}

Theorem 2. For system (6), at time $k$, if (59) has feasible solution, then (59) is solvable at time $k+1$ and system (37) is exponentially stable.

Proof. Denote the solution of (59) at time $k$ as follows:

$$
\left\{v_{s}^{*}(k)_{s=0}^{N-1}, \lambda_{s}^{*}(k)_{s=0}^{N-1}\right\} \text {. }
$$

We will prove, if one takes the following solutions:

$$
\begin{aligned}
v_{s}(k+1)_{s=0}^{N-2} & =v_{s+1}^{*}(k)_{s=0}^{N-2}, \\
\lambda_{s}(k+1)_{s=0}^{N-2} & =\lambda_{s+1}^{*}(k)_{s=0}^{N-2}, \\
v_{s}(k+1)_{s=N-1}^{\infty} & =0, \\
\lambda_{s}(k+1)_{s=N-1}^{\infty} & =\lambda(k),
\end{aligned}
$$

(59) is solvable at time $k+1$. Thus, one has $u_{s}(k+1)=u_{s+1}(k), \quad x_{s}(k+1)=x_{s+1}(k), \quad \xi_{s}^{(j)}(k+1)=$ $\xi_{s+1}^{(j)}(k)$, and $s \geq 0$.

Then, (52)-(55) hold at time $k+1$. In summary, (59) is feasible at time $k+1$.

Let $E=\gamma \widetilde{Q}^{-1}$. Consider a candidate Lyapunov function as

$$
V(\xi(k))=\sum_{s=0}^{N-1} \lambda_{s}^{*}(k)+\left\|x_{N}(k)\right\|_{E}^{2}
$$

In terms of (61), one has

$$
\begin{array}{r}
V(\xi(k+1))=\sum_{s=0}^{N-1} \lambda_{s}^{*}(k+1)+\left\|x_{N}(k+1)\right\|_{E}^{2} \\
\leq \sum_{s=1}^{N-1} \lambda_{s}^{*}(k)+\lambda(k)+\left\|x_{N+1}(k)\right\|_{E}^{2} .
\end{array}
$$

By exploiting (40), one has

$$
\begin{aligned}
& \left\|x_{N}(k+1)\right\|_{E}^{2}-\left\|x_{N}(k)\right\|_{E}^{2} \\
\leq- & \left(\left\|x_{N}(k)\right\|_{\mathscr{Q}}^{2}+\left\|u_{N}(k)\right\|_{\mathscr{R}}^{2}\right),
\end{aligned}
$$

then $V(\xi(k+1)) \leq V(\xi(k))$.

Hence, system (37) is exponentially stable.
Remark 4. In [26], the predictive control input $u_{s}(k)=K x_{\tau+s}(k)+v_{s}(k)$ are implemented to the abovementioned system. The $x_{\tau+i}(k)$ is uncertain, which is hard to construct $u_{N}(k+1)$. Therefore, the recursive feasibility is lost in theory.

Remark 5. In this paper, the control scheme is divided into two parts: offline robust controller synthesis and online robust receding horizon. Offline, the controller is designed to reduce the computation burden. Because the augmented state space model is used to handle the unstable system matrices, the computation burden is huge. Online, the free control moves are introduced into the online optimization problem to improve the control performance. This is the significance of the proposed control scheme.

4.3. Simulation Examples. In this section, we give two examples to demonstrate the effectiveness of the proposed approach. Example 1 is a numerical example, and Example 2 is an application-oriented example.

Example 1. The example is adapted from [31]. The system with polytopic uncertainty is

$$
\begin{aligned}
A(k) & =\left[\begin{array}{cc}
0.4 & 0.2 \\
\rho(k) & 0.4
\end{array}\right], \\
B & =\left[\begin{array}{l}
1 \\
0
\end{array}\right], \\
A_{\mu}(k) & =\beta_{\mu} A(k), \quad \mu=0,1,2,3, \\
B_{\tau}(k) & =\alpha_{\tau} B, \quad \tau=0,1,2,3,
\end{aligned}
$$

where the uncertainty parameter $\rho(k) \in[0.4,1.2]$. The input constraint $v=2, u(-3)=u(-2)=0$, and $u(-1)=0.2$, and there is no constraint for the system state. $\mathbb{Q}=I$ and $\mathscr{R}=1$.

Algorithm 1 in this paper is denoted by Algorithm 1, Algorithm of type-III in [31] without free control moves is denoted by IJC 2010. The comparisons between the Algorithm 1 and IJC 2010 are reported in this section. The online receding horizon $N=2$.

Case 1. $\beta_{3}=\alpha_{0}=0, \quad \beta_{0}=\beta_{1}=\beta_{2}=\beta=0.37$, and $\alpha_{1}=\alpha_{2}=\alpha_{3}=1$.

Figures 1 and 2 depict the state responses of $x_{1}$ and $x_{2}$, respectively, for $\beta=0.37$. From Figures 1 and $2, x_{1}$ and $x_{2}$ fluctuate as time goes and tend to the zero as $k$ increases; therefore, the proposed controller stabilizes the closed-loop systems with unstable matrices. Besides, the fluctuation range of IJC 2010 is stronger than Algorithm 1. On the contrary, Algorithm 1 with free control moves makes the states of the system smoother.

Figure 3 depicts the evolution of $\gamma(k)$. It is shown that $\gamma(k)$ monotonically decrease with the increase of $k$. Compared with IJC 2010, a smaller upper of cost function and a more quick convergence rate are achieved for Algorithm 1. Therefore, the control performance is improved by introducing free control moves for Algorithm 1. 
(offline) Choose the augmented state $\xi(0)$ and polytopic vertices, solve the offline optimization problem (35) to obtain the controller gain $K$.

(online)

(1) Calculate $\xi(k)$ by (6);

(2) Compute the optimization problem (59), obtain the free control moves $\left\{v_{0}(k), v_{1}(k), \ldots, v_{N-1}(k)\right\}$;

(3) Implement $u(k)=K \xi(k)+v_{0}(k)$;

(4) At time $k+1$, return to (1).

Algorithm 1: $N$ free control move approach of multiple time delay robust RHC.

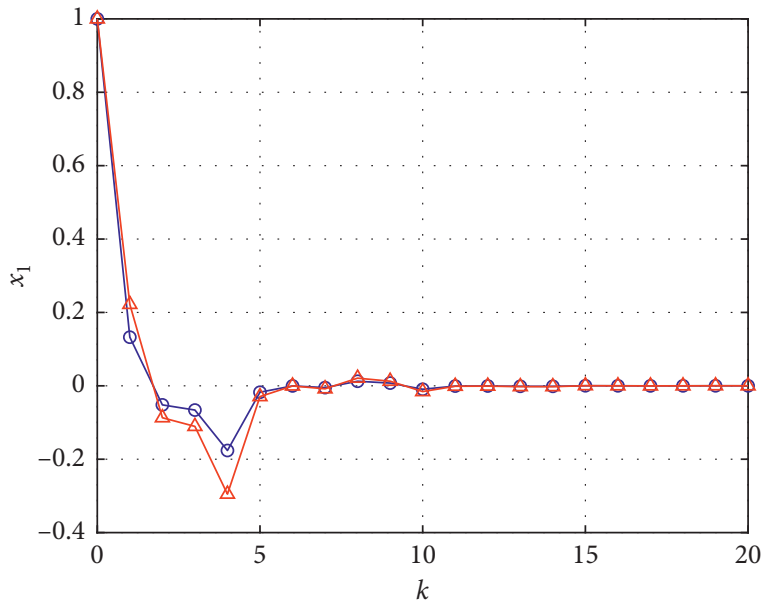

๑ Algorithm 1

$\triangle \mathrm{IJC} 2010$

Figure 1: The state response of $x_{1}$ for $\beta=0.37$.

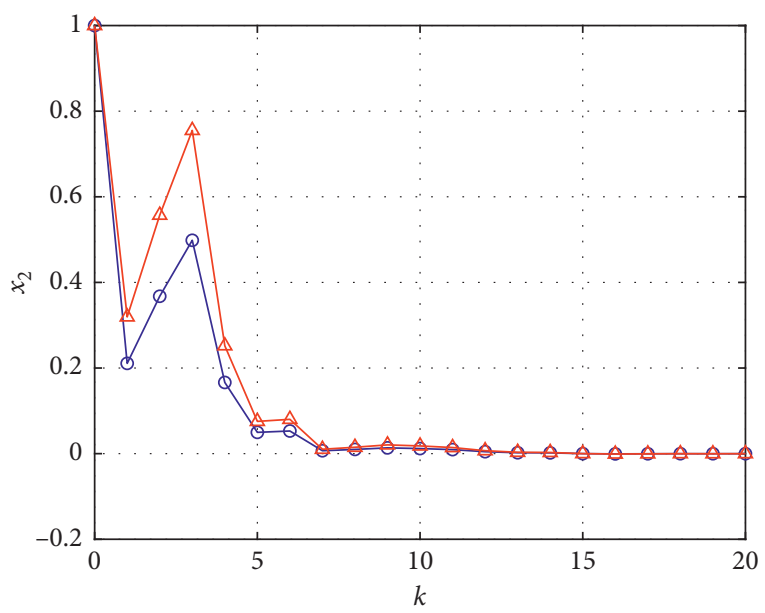

$$
\begin{aligned}
& \_ \text {Algorithm 1 } \\
& \triangle \text { IJC2010 }
\end{aligned}
$$

FIgURe 2: The state response of $x_{2}$ for $\beta=0.37$.

Figure 4 depicts the control inputs. From Figure 4, we have the following results: (1) the input constraints are both satisfied for the two approaches. (2) The initial value of control input for Algorithm 1 is -0.54 , while IJC 2010 is -0.38 . Moreover, the maximum value of control input for

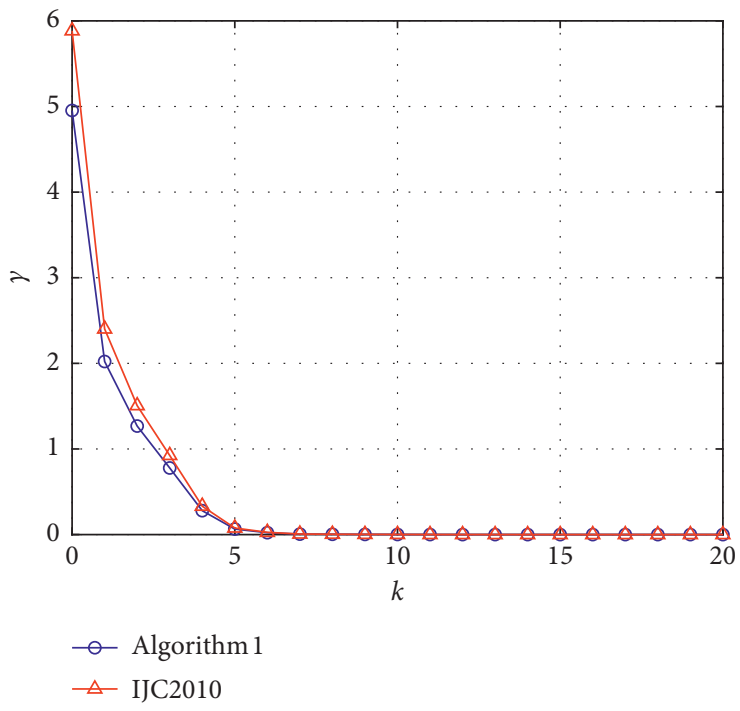

FIgURE 3: Evolution of $\gamma$ for $\beta=0.37$.

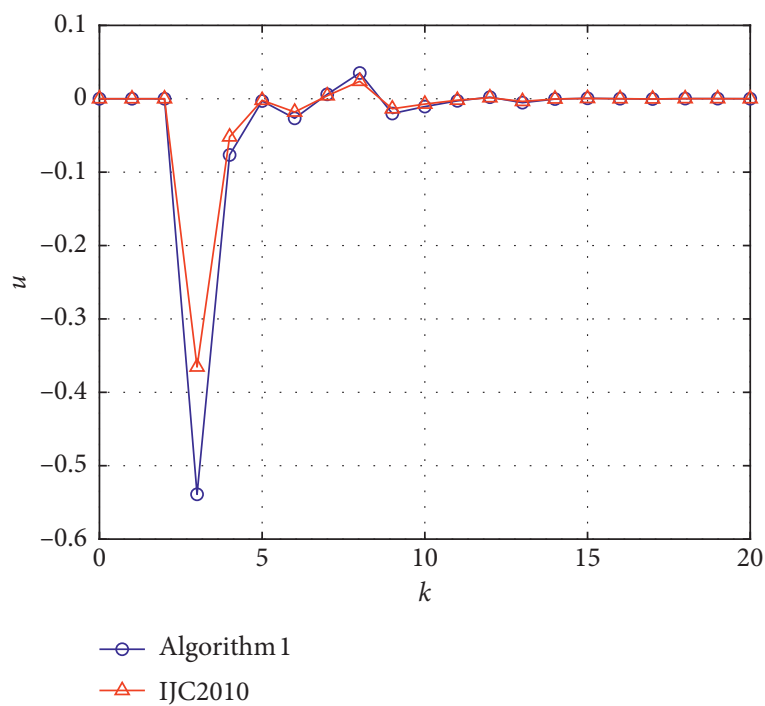

Figure 4: Control input signal for $\beta=0.37$.

Algorithm 1 is larger than IJC 2010. Therefore, the input range of the Algorithm 1 is wider than IJC 2010. (3) Algorithm 1 and IJC 2010 both converge to the neighborhood of 0 . Algorithm 1 does not reduce the speed of convergence while amplifying the control signal. 
Hence, Algorithm 1 has a better control performance and stronger robustness.

Case 2. $\beta_{2}=\beta_{3}=\alpha_{0}=\alpha_{1}=0, \quad \beta_{0}=\beta_{1}=\beta=0.86$, and $\alpha_{2}=\alpha_{3}=1$.

The state responses of $x_{1}$ and $x_{2}$, are depicted in Figures 5 and 6, respectively. Similar to Figures 1 and 2, all states converge to zero as time goes by, which demonstrates that the closed-loop system with unstable matrices is stable.

The evolution of $\gamma(k)$ is shown in Figure 7. It is clear that $\gamma(k)$ is monotonically decreasing for both algorithms, and the cost function of Algorithm 1 sharply reduces. Thus, Algorithm 1 with free control moves has a better control performance.

The control inputs are depicted in Figure 8. According to Figure 8, for Algorithm 1 and IJC 2010, the input constraints are guaranteed. Moreover, the initial value of the control input for Algorithm 1 is -1.2 , while IJC 2010 is -0.59 . Compared with IJC 2010, the maximum value of the control input for Algorithm 1 is larger. Therefore, a wider input range is achieved for Algorithm 1.

Hence, Algorithm 1 with free control moves not only can stabilize the system with unstable system matrices but also improve the control performance.

To further validate the advantage of the proposed algorithm with free control moves, the other two cases are considered.

Case 3. $\beta_{3}=\alpha_{0}=0, \quad \alpha_{1}=\alpha_{2}=\alpha_{3}$, and $\beta_{0}=\beta_{1}=\beta_{2}=$ $\beta=0.28$.

Case 4. $\beta_{1}=\beta_{2}=\beta_{3}=\alpha_{0}=\alpha_{1}=\alpha_{2}=0, \beta_{1}=1.56$, and $\alpha_{3}=1$.

For $\beta=0.28$, the control inputs are depicted in Figure 9, and the evolution of $\gamma(k)$ are shown in Figure 10. For $\beta=0.28$, the control inputs and the evolution of $\gamma(k)$ are shown in Figures 11 and 12, respectively. Similar to Case 1 and Case 2, simulation results come to the same conclusions for Case 3 and Case 4. These simulation results demonstrate the advantage of the proposed algorithm.

In summary, the following conclusions are obtained from the figures.

Firstly, controller (5) can stabilize the system with unstable system matrices and more than one input delay, which is consistent with "Theorem 2".

Secondly, Algorithm 1 has a more aggressive input and a better control performance. Therefore, it is very necessary to apply the receding horizon policy with free control moves to the systems with unstable system matrices and more than one input delay. Therefore, it is very necessary to apply the receding horizon policy with free control moves to the systems with unstable system matrices and more than one input delay.

Example 2. An application-oriented example is adopted to verify the proposed result. The example is adapted from [23]. The urban water system is established based on the underground water network. The details can refer to [23]. The system parameters are as follows:

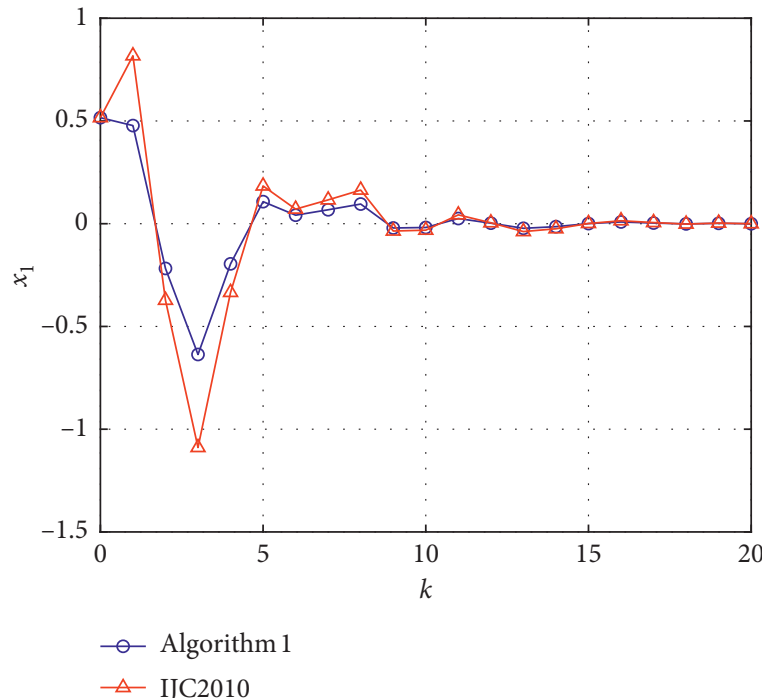

FIgURE 5: The state response of $x_{1}$ for $\beta=0.86$.

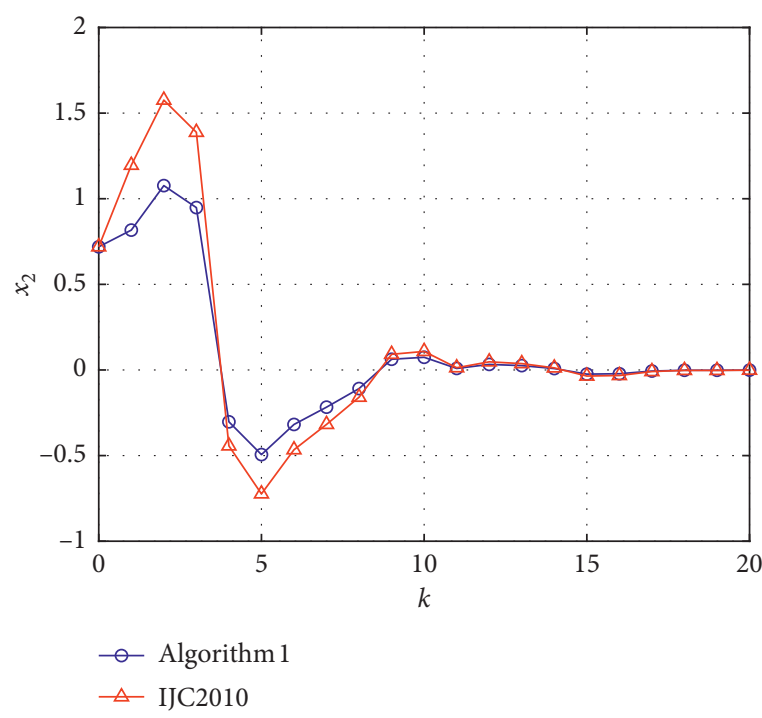

FIgURE 6: The state response of $x_{2}$ for $\beta=0.86$.

$$
\begin{aligned}
& A_{0}(1)=\left[\begin{array}{ll}
0.40 & 0.62 \\
0.62 & 0.55
\end{array}\right], \\
& A_{0}(2)=\left[\begin{array}{ll}
0.41 & 0.65 \\
0.65 & 0.60
\end{array}\right], \\
& A_{1}(1)=\left[\begin{array}{ll}
0.40 & 0.37 \\
0.35 & 0.45
\end{array}\right], \\
& A_{1}(2)=\left[\begin{array}{ll}
0.42 & 0.38 \\
0.35 & 0.46
\end{array}\right], \\
& B_{0}(1)=\left[\begin{array}{ll}
0.01 & 0.02 \\
0.02 & 0.03
\end{array}\right], \\
& B_{0}(2)=\left[\begin{array}{ll}
0.03 & 0.04 \\
0.03 & 0.03
\end{array}\right] .
\end{aligned}
$$




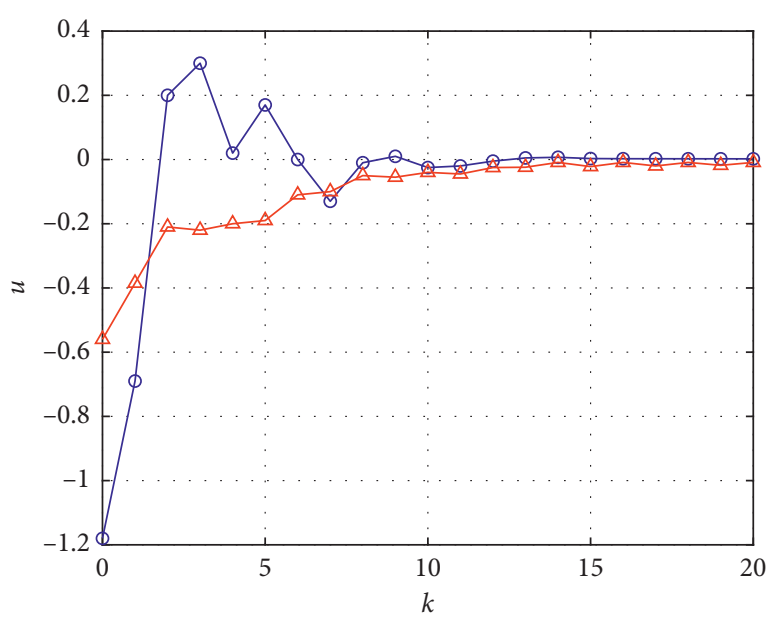

○- Algorithm 1

$\triangle$ IJC2010

Figure 7: Control input signal for $\beta=0.86$.

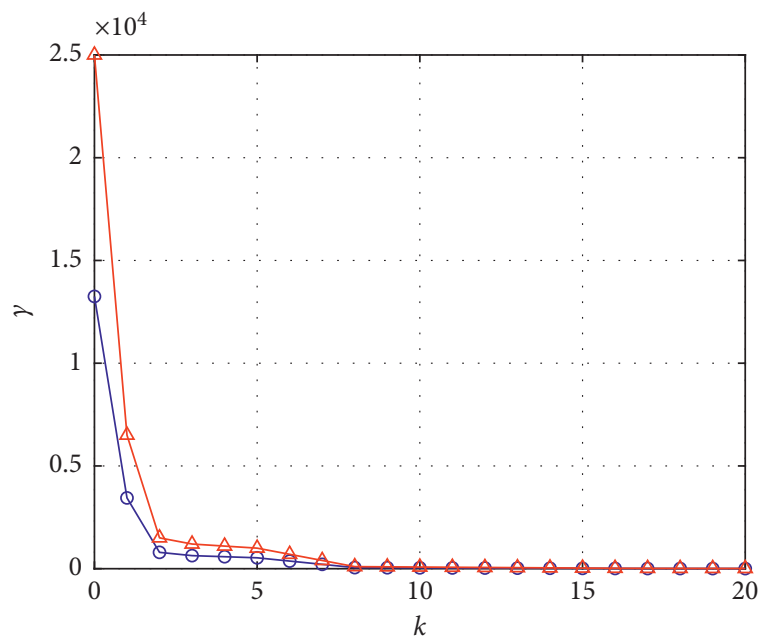

- Algorithm 1

$\triangle \mathrm{IJC} 2010$

FIgURE 8: Evolution of $\gamma$ for $\beta=0.86$.

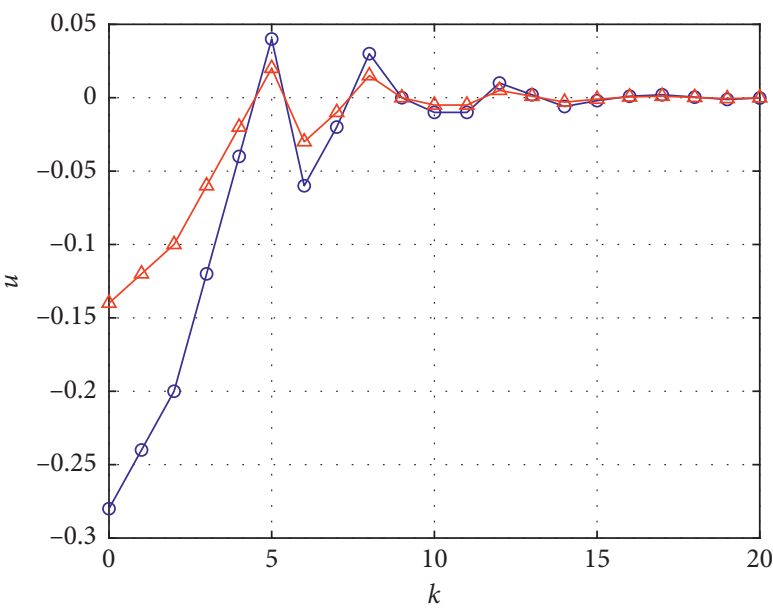

- Algorithm 1

$\triangle$ IJC2010

Figure 9: Control input signal for $\beta=0.28$.

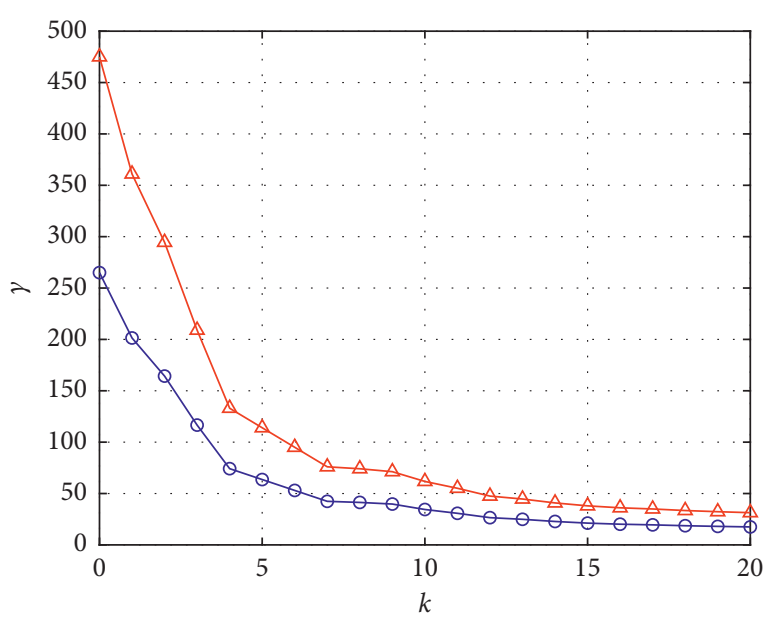

- Algorithm 1

$\triangle$ IJC2010

Figure 10: Evolution of $\gamma$ for $\beta=0.28$.

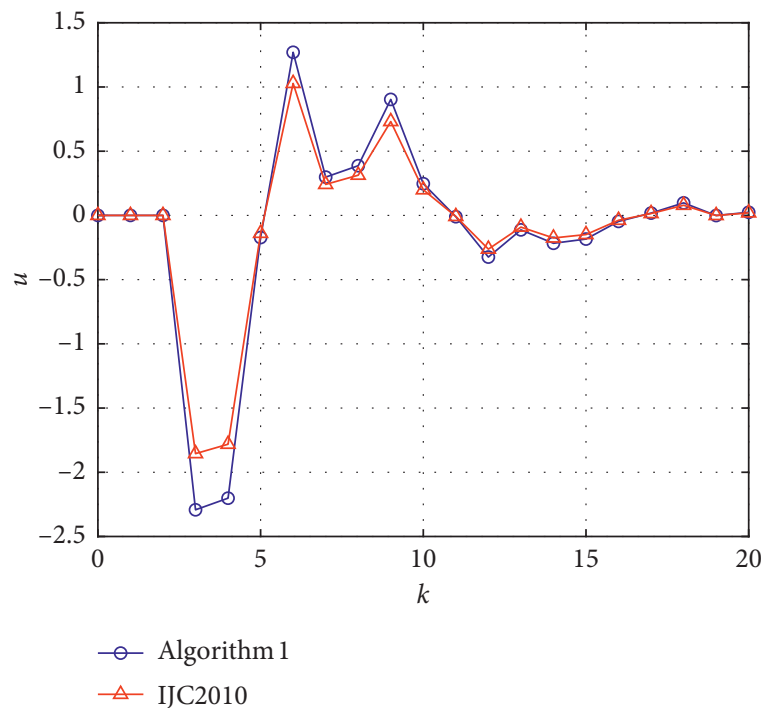

Figure 11: Control input signal for $\beta=1.56$.

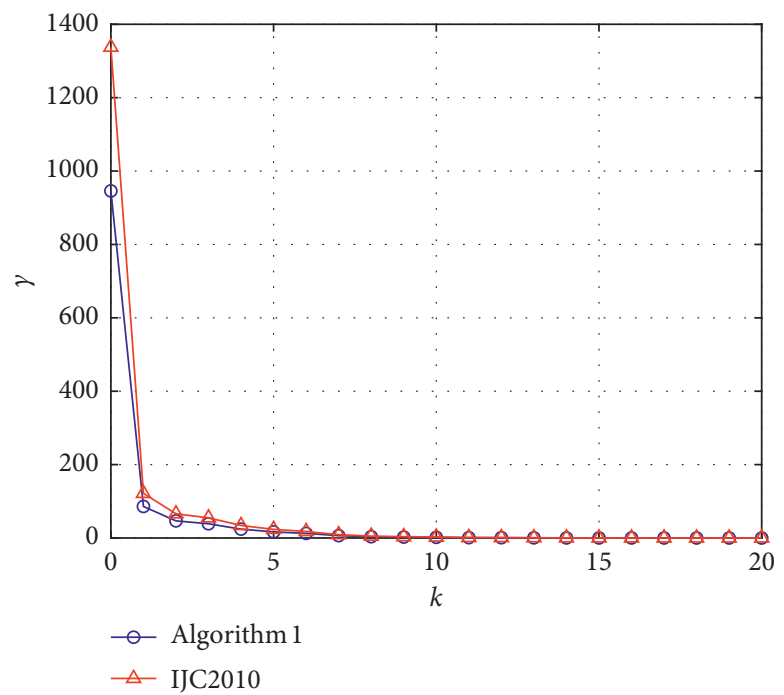

FIgURE 12: Evolution of $\gamma$ for $\beta=1.56$. 


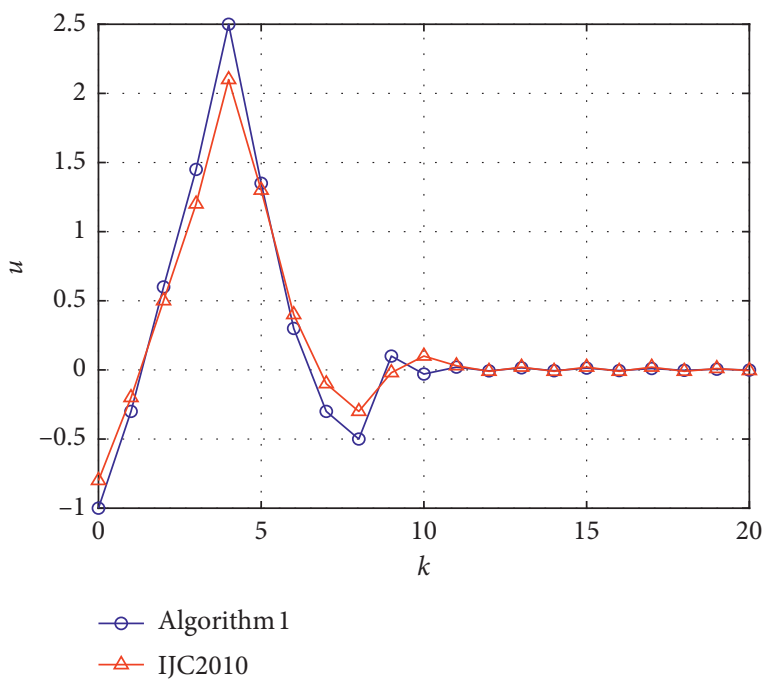

FIgURE 13: Control input signal.

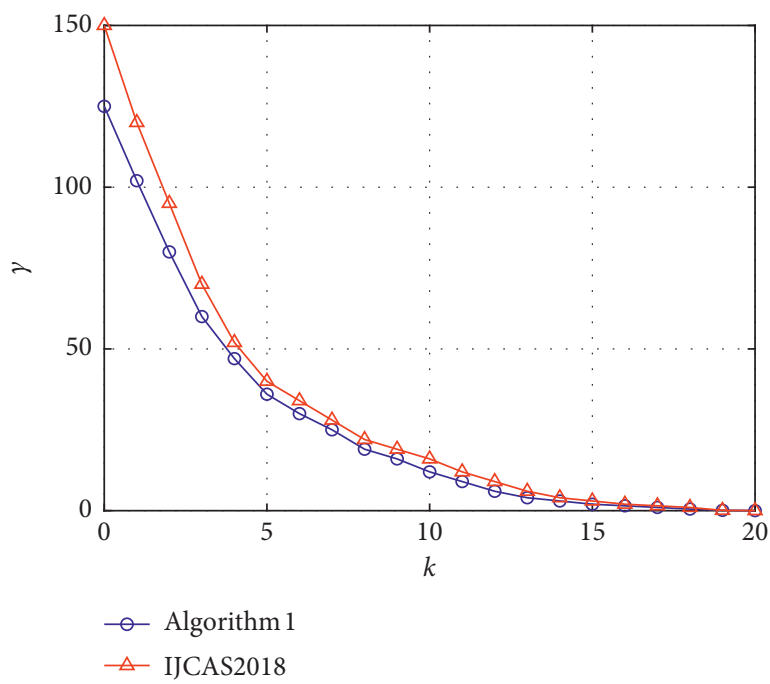

FIgURE 14: Evolution of $\gamma$.

Initial state $x(k)=(21)^{\mathrm{T}}, u(-1)=0.2$, input constraint $\nu=1.8$, state constraint $\phi=1.5$. $Q=0.5$, and $\mathscr{R}=0.5$, and the online receding horizon $N=3$.

In [23], the MPC control scheme is without the free control moves in the controller designing. The algorithm of [23] is denoted by IJCAS 2018. The comparisons between Algorithm 1 and IJCAS 2018 are given to show the effectiveness of the proposed approach.

For Algorithm 1 and IJCAS 2018, the control input $u$ is shown in Figure 13, and the evolution of $\gamma$ is depicted in Figure 14.

The analysis of simulation results is the same as Example 1. The simulation results also reflect that Algorithm 1 has a better control performance.

\section{Conclusions}

In this paper, the free control move approach is generalized to the systems with unstable system matrices and more than one input delay. By designing a new local feedback controller based on the augmented state, the recursive feasibility and closed-loop stability are guaranteed, where the local feedback gain is designed offline and the free control moves are minimized by online optimization problem. In this paper, the time delay is a constant and disturbance is not considered. Therefore, the free control move approach will be further applied to the system with time-varying time delays and disturbance in the future works.

\section{Data Availability}

The data used to support the findings of this paper have been deposited in the Taylor and Francis repository (DOI: 10. 1080/00207179/2010.498058).

\section{Conflicts of Interest}

The authors declare that there are no conflicts of interest regarding the publication of this paper.

\section{Acknowledgments}

This work was supported by the National Key R\&D Program of China (no. 2018YFB1700104) and NSFC-Zhejiang Joint Fund for the Integration of Industrialization and Informatization (no. U1809207).

\section{References}

[1] Z. Wan and M. V. Kothare, "An efficient off-line formulation of robust model predictive control using linear matrix inequalities," Automatica, vol. 39, no. 9, pp. 837-846, 2003.

[2] S. Li, Y. Zhang, and Q. Zhu, "Nash-optimization enhanced distributed model predictive control applied to the shell benchmark problem," Information Sciences, vol. 170, no. 2-4, pp. 329-349, 2005.

[3] Q. Pang, T. Zou, Q. Cong, and Y. Wang, "Constrained model predictive control with economic optimization for integrating process," The Canadian Journal of Chemical Engineering, vol. 93, no. 8, pp. 1462-1473, 2015.

[4] N. Chen, J. Dai, X. Zhou, Q. Yang, and W. Gui, "Distributed model predictive control of iron precipitation process by goethite based on dual iterative method," International Journal of Control, Automation and Systems, vol. 17, no. 5, pp. 1233-1245, 2019.

[5] R. Goebel and S. V. Raković, "Set-valued and Lyapunov methods for MPC," in Handbook for Model Predictive Control (Control Engineering), Birkhäuser, Cham, Switzerland, 2019.

[6] H. Li and Y. Shi, "Networked min-max model predictive control of constrained nonlinear systems with delays and packet dropouts," International Journal of Control, vol. 86, no. 4, pp. 610-624, 2013.

[7] H.-T. Zhang, Z. Cheng, G. Chen, and C. Li, "Model predictive flocking control for second-order multi-agent systems with input constraints," IEEE Transactions on Circuits and Systems I: Regular Papers, vol. 62, no. 6, pp. 1599-1606, 2015.

[8] Z. Yan, J. Wang, S. Wang, S. Li, D. Wang, and W. Sun, "Model predictive control method of simulated moving bed chromatographic separation process based on subspace system identification," Mathematical Problems in Engineering, vol. 2019, Article ID 2391891, 24 pages, 2019. 
[9] K. Yu, X. Xu, Q. Liang et al., "Model predictive control for connected hybrid electric vehicles," Mathematical Problems in Engineering, vol. 2015, Article ID 318025, 15 pages, 2015.

[10] P. Selvaraj, R. Sakthivel, S. Marshal Anthoni, M. Rathika, and M. Yong-Cheol, "Dissipative sampled-data control of uncertain nonlinear systems with time-varying delays," Complexity, vol. 21, no. 6, pp. 142-154, 2015.

[11] X. Jia, D. Zhang, X. Hao et al., "Fuzzy tracking control for nonlinear networked control systems in T-S fuzzy model," IEEE Transactions on Systems Man \& Cybernetics Part B Cybernetics, vol. 39, no. 4, pp. 1073-1079, 2009.

[12] P. Selvaraj, O. M. Kwon, and R. Sakthivel, "Disturbance and uncertainty rejection performance for fractional-order complex dynamical networks," Neural Networks, vol. 112, pp. 73-84, 2019.

[13] E. Camacho and C. Bordons, Model Predictive Control, Springer-Verlag, New York, NY, USA, 2004.

[14] W. Qin, G. Liu, L. Wang, and Z. Zheng, "Memory state feedback RMPC for multiple time-delayed uncertain linear systems with input constraints," Mathematical Problems in Engineering, vol. 2014, Article ID 409863, 9 pages, 2014.

[15] D.-F. He, H. Huang, and Q.-X. Chen, "Quasi-min-max MPC for constrained nonlinear systems with guaranteed input-tostate stability," Journal of the Franklin Institute, vol. 351, no. 6, pp. 3405-3423, 2014.

[16] L. Kong and J. Yuan, "Disturbance-observer-based fuzzy model predictive control for nonlinear processes with disturbances and input constraints," ISA Transactions, vol. 90, no. 1, pp. 74-88, 2019.

[17] Z. Wan and M. V. Kothare, "Robust output feedback model predictive control using off-line linear matrix inequalities," Journal of Process Control, vol. 12, no. 7, pp. 763-774, 2002.

[18] D. Li, Y. Xi, and P. Zheng, "Constrained robust feedback model predictive control for uncertain systems with polytopic description," International Journal of Control, vol. 82, no. 7, pp. 1267-1274, 2009.

[19] A. Gautam, Y. Chu, and Y. Chai, "Optimized dynamic policy for receding horizon control of linear time-varying systems with bounded disturbances," IEEE Transactions on Automatic Control, vol. 57, no. 1, pp. 973-987, 2012.

[20] T. Shi, H. Su, and J. Chu, "An improved model predictive control for uncertain systems with input saturation," Journal of the Franklin Institute, vol. 350, no. 9, pp. 2757-2768, 2013.

[21] X. Jia, L. Li, D. Zhang, X. Chi, and X. Fan, "Exponential stabilization of dual-rate control system: a switched system approach," in Proceedings of the American Control Conference, Washington, DC, USA, June 2013.

[22] S. C. Jeong and P. Park, "Constrained MPC algorithm for uncertain time-varying systems with state-delay," IEEE Transactions on Automatic Control, vol. 50, no. 2, pp. 257263, 2005.

[23] J. Zhang, H. Yang, M. Li, and Q. Wang, "Robust model predictive control for uncertain positive time-delay systems," International Journal of Control Automation and Systems, vol. 17, no. 2, pp. 307-318, 2019.

[24] J. Lu, D. Li, and Y. Xi, "Probability-based constrained MPC for structured uncertain systems with state and random input delays," International Journal of Systems Science, vol. 44, no. 7, pp. 1354-1365, 2013.

[25] M. V. Kothare, V. Balakrishnan, and M. Morari, "Robust constrained model predictive control using linear matrix inequalities," Automatica, vol. 32, no. 10, pp. 1361-1379, 1996.

[26] B. Ding and B. Huang, "Constrained robust model predictive control for time-delay systems with polytopic description,"
International Journal of Control, vol. 80, no. 4, pp. 509-522, 2007.

[27] Y. Lu and Y. Arkun, "Quasi-min-max MPC algorithms for LPV systems," Automatica, vol. 36, no. 4, pp. 527-540, 2000.

[28] A. Casavola, D. Famularo, and G. Franzè, "Robust constrained predictive control of uncertain norm-bounded linear systems," Automatica, vol. 40, no. 12, pp. 1865-1876, 2004.

[29] B. Kouvaritakis, J. A. Rossiter, and J. Schuurmans, "Efficient robust predictive control," IEEE Transactions on Automatic Control, vol. 45, no. 8, pp. 1545-1549, 2000.

[30] J. Schuurmans and J. A. Rossiter, "Robust predictive control using tight sets of predicted states," IEE Proceedings-Control Theory and Applications, vol. 147, no. 1, pp. 13-18, 2000.

[31] B. Ding, "Robust model predictive control for multiple time delay systems with polytopic uncertainty description," International Journal of Control, vol. 83, no. 9, pp. 1844-1857, 2010. 